\title{
COVID-19: Oral Manifestations-A Dentist's Perspective
}

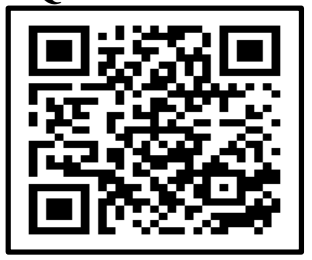

\section{RUHEE SANGHA, SUMANJIT² id, RAMANDEEP SANDHU³, MOHIT BANSAL*4iD, SIMRAN5iD}

COVID-19 is a global pandemic disease which has crippled health care system and economics worldwide. According to WHO, an acute respiratory infection, fever and cough are the most valid diagnostic clinical features. Some common orofacial manifestations of this viral infection may contribute to early diagnosis of covid-19 infection. Oral manifestations include olfactory and gustatory disturbances, dry mouth, facial pain, vesicobullous lesions such as erosion, pustule, macule, papule, plaque, pigmentation and depapilliated tongue with whitish areas, haemorrhagic crust, necrosis, swelling and erythema. The most common sites of involvement in descending order are tongue (38\%), labial mucosa $(26 \%)$, and palate $(22 \%)$. Oral lesions are symptomatic in $68 \%$ of the cases who test positive for SARS Co-V. Lack of oral hygiene, opportunistic infections, stress, immunosuppression, vasculitis, and hyper-inflammatory response secondary to COVID-19 are the most important predisposing factors for onset of oral lesions in COVID-19 patients.

KEYWORDS: COVID-19, Dysgeusia, Oral, Ulcer

\section{INTRODUCTION}

Corona virus disease 2019 (COVID-19) is an infection caused by Severe Acute Respiratory Syndrome (SARS) CoV- 2 which is the seventh type belonging to the coronavirus family to affect human beings. ${ }^{1}$ The novel coronavirus (COVID-19) pandemic plaguing the entire world originated from Wuhan city, China in the month of December 2019. This SARS-CoV-2 is a respiratory zoonotic disease having both bats and pangolins as the most probable origin and intermediate host. ${ }^{2,3}$ Initially, the identification and sequencing of the virus responsible for COVID-19 led to the conclusion that it is a novel Coronavirus ( $\mathrm{CoV})$ that shared an astonishing $88 \%$ sequence identity with two bat-derived SARS-like $\mathrm{CoV}$, suggesting its origin in bats.

Further testing and full-length genome sequencing revealed that the 2019-nCoV shares $79.5 \%$ sequence identity with SARS-CoV; and consequently, protein sequence analysis divulged that the virus belonged to a class of SARS-related coronaviruses. The $2019-\mathrm{nCoV}$ is believed to spread through close person-to-person contact of about 2 metres, a distance at which the respiratory droplets from an infected person either asymptomatic or symptomatic person (who coughs, sneezes, or speaks) can spread to other people who do not have adequate barriers (Proper distancing and masks). Another probable route of transmission occurs indirectly, when saliva droplets fall on other surfaces, such as the ground and objects made of different materials, and people come into contact with them. ${ }^{4}$ COVID - 19 has an incubation period of 1 to 15 days with most ranging from 3 to 7 days. ${ }^{5}$ Upon global protest, the World health Organization (WHO) declared COVID-19 as a global pandemic.

\section{SARS COV AND ACE INHIBITORS}

As with any infection, the entry of the virus in host cells is the first step in the initiation of the infection; with the presence of a spike glycoprotein on the viral envelope of the coronavirus binding to specific receptors on the membrane of host cells. Laboratory based studies have documented that ACE2 is the specific functional receptor for SARS-CoV. It was further revealed that this virus can enter ACE2 expressing cells, as the virus did not affect cells without $\mathrm{ACE} 2$ receptors or other cells expressing coronavirus receptors [which include aminopeptidase $\mathrm{N}$ and dipeptidyl peptidase 4 $\left.\left(\mathrm{DPP}_{4}\right)\right] .{ }^{6,7}$

The receptor-binding domain of the spike glycoprotein was reported to bind itself to the tip of subdomain I of ACE2. The fusion of the virus to the cell membrane of the host cell is activated after binding, which leads to viral RNA being subsequently released into the 
cytoplasm, and thus manifesting infection. In this process, a few transmembrane proteinases [disintegrin and metallopeptidase domain 17 (ADAM17) particularly], transmembrane protease serine 2 (TMPRSS2)], TNF-converting enzyme and proteins (which include vimentin and clathrin) are documented to be involved in the processes of binding and membrane fusion.

The ACE2 receptor is expressed in differing degrees in all human organs and in the respiratory system, it is mainly expressed in the type II alveolar epithelial cells of the oral and nasal mucosa and nasopharynx, indicating that the lungs are in fact the primary target of SARS viruses. In addition, the ACE2 receptor is also reported to be highly expressed in myocardial cells, proximal tubule cells of the kidney, the urothelial cells of the bladder, and abundantly expressed on the enterocytes of the small intestine. The virus, which is cell-free, and is macrophage phagocytosis-associated might possibly spread from the lungs to other vital organs with a high ACE2 expression through blood circulation. Figure 1 depicts the diagrammatic representation of virus attaching to host cell.

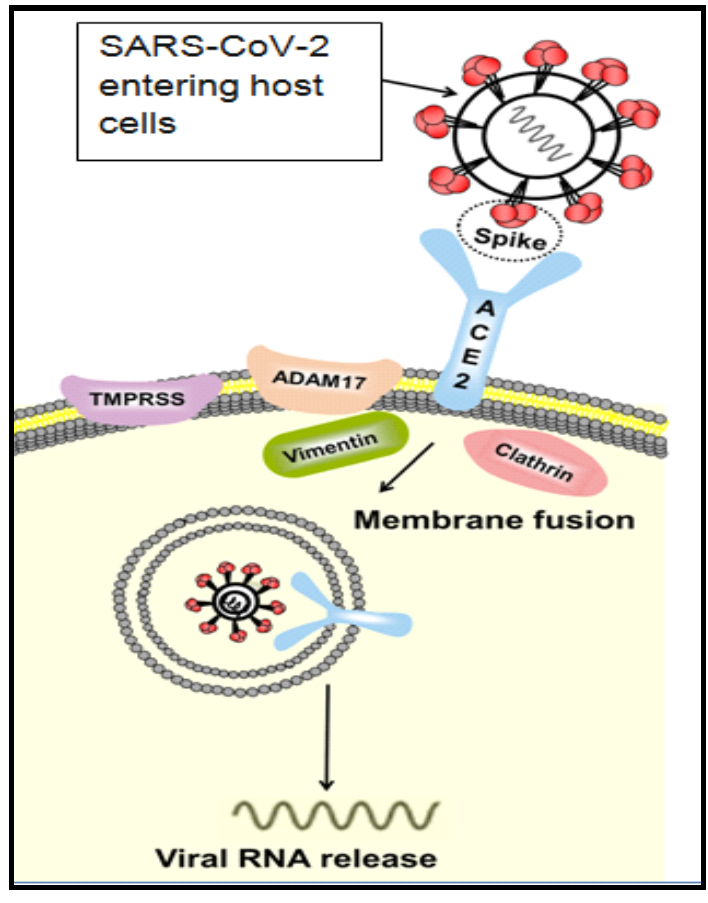

Figure 1. Diagrammatic Representation of Virus Attaching to Host Cell

ACE-2 was expressed in the oral cavity more prominently in epithelial cells of the tongue as compared to other oral sites such as gingival and buccal mucosa. ${ }^{8}$ In clinical studies, it was found to be strongly expressed in taste buds of rats. Furthermore, angiotensin II produced in taste buds of mice demonstrated an essential role altering taste sensitivity. Since ACE2 is found to degrade the Ang II receptors, which might lead to possible accumulation of Ang II in taste buds during COVID-19 infection and this mechanism possibly explains the reason behind the reported dysgeusia among patients.

\section{CNS INVASION ${ }^{6}$}

The CNS invasion of the virus is shown in figure 2.

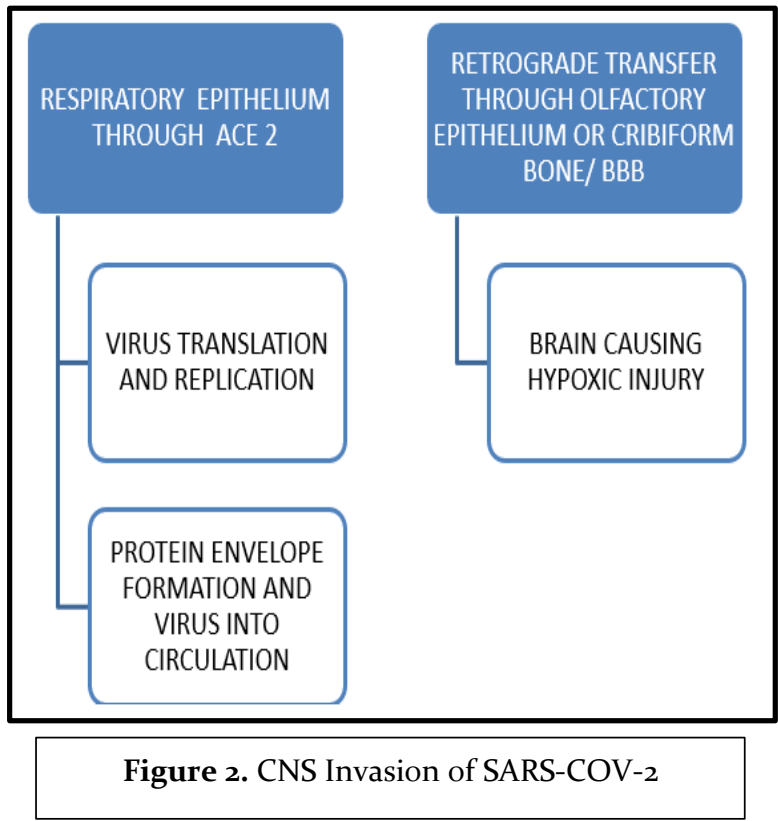

\section{MECHANISM OF SARS-CoV-2 IN ORAL CAVITY7}

The mechanism of action of SARS-CoV-2 on the oral cavity is shown in figure 3.

\section{ORAL MANIFESTATIONS OF SARS-CoV-2}

Olfactory Dysfunction: According to Biadsee et $(2020)^{8}, 67 \%$ of patients report olfactory dysfunction during the disease. These olfactory impairments can be classified into two main categories: the first being conductive losses stemming from obstruction of nasal passages and the second: sensorinueral causes from damage to the olfactory nueroepithelium which are most often attributed to post viral olfactory loss.

Ulceration in The Oral Cavity: According to a review by Behzad et al., oral manifestations of patients affected 


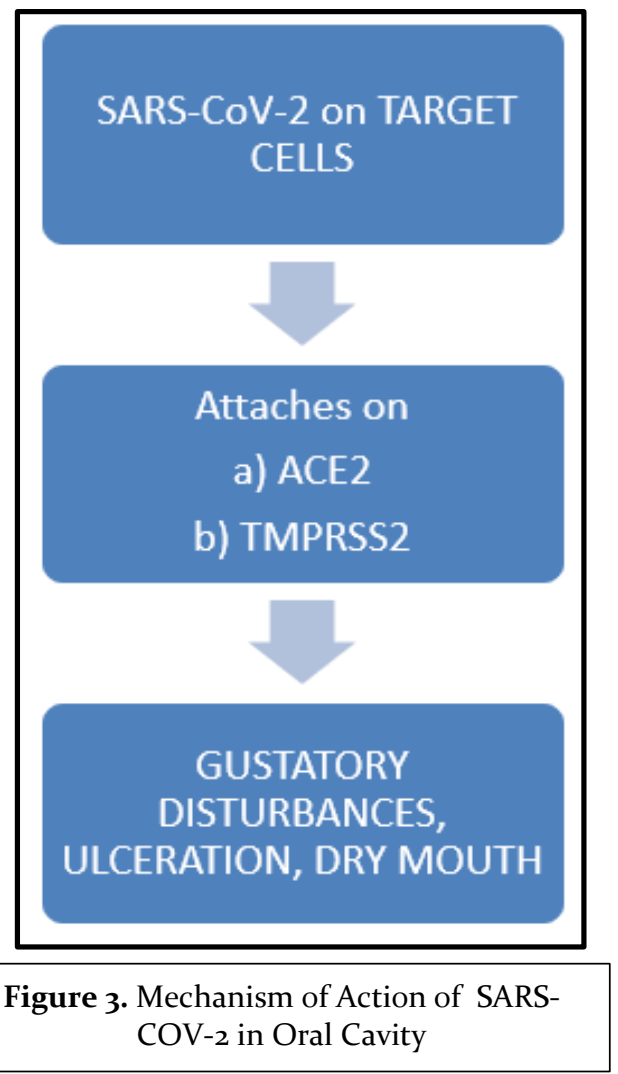

with COVID-19 included ulcer, erosion, bulla, vesicle, pustule, fissured or depapillated tongue, macule, papule, plaque, pigmentation, halitosis, whitish areas, hemorrhagic crust, necrosis, petechiae, swelling, erythema, and spontaneous bleeding. The most common sites of involvement in descending order were tongue $(38 \%)$, labial mucosa $(26 \%)$, palate $(22 \%)$, gingiva $(8 \%)$, buccal mucosa $(5 \%)$, oropharynx $(4 \%)$,and tonsil (1\%). Suggested diagnoses of the lesions were aphthous stomatitis, herpetiform lesions, candidiasis, vasculitis, Kawasaki-like, EM like mucositis, drug eruption, necrotizing periodontal disease, bullosa-like, angular cheilitis, atypical Sweet syndrome, and MelkersonRosenthal syndrome. The oral lesions in approximately $68 \%$ of the patients were reported to be symptomatic (painful, burning sensation, or pruritus) and were nearly equally distributed among both genders ( $49 \%$ female, $51 \%$ male). The latent time between appearance of oral lesions was between 4 days before and up to 12 weeks after the onset of systemic symptoms among patients. ${ }^{9}$

Biadsee and colleagues demonstrated that $7 \%$ of the patients with RT-PCR positive test had plaque-like changes on the dorsum of tongue. Also, swelling of oral cavity including palatal, lingual, and gum) was reported by $8 \%$ of the patients. In addition, the appearance of oral lesions was simultaneously observed with a loss of taste and smell in patients with more severe and disseminated oral lesions reported among older patients. ${ }^{8}$

Stress and immunosuppression associated with COVID19 and drug eruption, vasculitis, or thrombotic vasculopathy secondary to COVID-19 were suggested as causes for development of ulcerative and erosive lesions and appearance of secondary herpetic gingivostomatitis. Oral lesions also included a painful, diffuse erythematous gingiva with necrosis of inter-papillary areas which suggested diagnosis of necrotizing periodontal disease as a result of bacterial co-infections. The lesions recovered after 5 days. ${ }^{9}$

Taste: Among patients, a true loss of taste is extremely rare, and is routinely preceded by the inability to perceive the odor of food due to olfactory dysfunction. ${ }^{10}$ Biadsee et al. reported that $25.8 \%$ patients with an impaired sense of smell and taste in the absence of other symptoms. The pooled eligible data for different taste disorders were $38 \%$ for dysgeusia, $35 \%$ for hypogeusia while ageusia had a prevalence of $24 \%$. A loss of smell or taste as a result of COVID-19 seems to last slightly longer as compared to various other upper respiratory infections. The median reported duration of loss of smell or taste among patients suffering from COVID-19 was 8 days.

Dry Mouth: There is a strong correlation found between burning mouth and alteration of taste. Since saliva functions as a solvent of taste and regulates the conditions of taste receptors, a change in the salivary flow can be potentially linked to an altered taste alteration among patients. Taste disorders involve the damage of gustatory papillae and taste buds caused by reduced saliva secretion. Since human salivary glands express ACE2 and TMPRSS2, salivary gland infection with SARS-CoV-2 would affect saliva secretion, thereby altering the tasting ability. ${ }^{11}$

The element zinc has been reported to increase the secretion of unstimulated and stimulated saliva in humans, and its deficiency is associated with COVID-19 as it may induce gustatory dysfunction through hyposalivation. The important functions of zinc not only include building the immune system and inflammatory response, but also involves the gustatory function at a level of taste buds and taste stimulus-transmitting nerves. It is also required for the subsequent regeneration and maintenance of taste cells and for zinc-metalloenzymes localized in taste buds. Taste 
disorders are improved by administrating zinc as zinc ionophore chloroquine which increases the cellular influx of zinc that inhibits SARS-CoV-2. A decreased zinc level is also favorable for the interaction of zincdependent metalloenzyme ACE2 with spike proteins of SARS-CoV-2 but an increased zinc level inhibits ACE2 expression. $^{12}$

Periodontal Tissue: Among periodontal tissues, ACE2, TMPRSS2, and furin have been documented to play an important and have an established role in the viral cell invasion and are expressed in the epithelium of the gingival sulcus and periodontal pocket. Hence, the virus potentially infects these epithelia and leads to a potentially adverse effect on periodontal tissues. The major periodontopathic bacteria Prevotella intermedia has been frequently detected among COVID-19 patients. On subsequent examination, the intraoral cavity of patients revelaed severe halitosis, generalized erythematous, edematous gingivae, and necrotic papillae (interdental) in both the maxillary and mandibular sextants on the labial side. ${ }^{13}$

Inflammation of Salivary Glands: Cases involving inflamed salivary glands have been reported among COVID-19 patients due to the expression of ACE2 in these glands. The virus can cause acute sialadenitis by binding to ACE2 receptors in the epithelia of the salivary glands and lead to lysing of the cells." The inflammatory damages to the acinar cells are subsequently repaired by fibroblast proliferation and fibrous connective tissue formation, this fibrous repair and hyperplasia may give rise to salivary gland hyposecretion and ductal stenosis, as a result of which, the salivary flow is reduced. ${ }^{14}$

\section{CONCLUSION}

SARS-CoV-2 may manifest with various combinations of symptoms. Aphthous-like lesions, gustatory disturbance, candidiasis, xerostomia and oral lesions of Kawasaki-like disease are the most common oral manifestations of COVID-19 disease. Lack of oral hygiene, opportunistic infections, stress, underlying diseases (diabetes mellitus, immunosuppression), trauma (secondary to intubation), vascular compromise, and hyper-inflammatory response secondary to COVID-19 might be the most important predisposing factors for the development of oral lesions in COVID-19 patients.

\section{REFERENCES}

1. Zhu N, Zhang D, Wang W, Li X, Yang B, Song J, et al. A novel coronavirus from patients with pneumonia in
China, 2019. N Engl J Med. 2020;382(8):727-33. https://doi.org/10.1056/NEJMoa2001017

2. Mackenzie JS, Smith DW. COVID-19: a novel zoonotic disease caused by a coronavirus from China: what we know and what we don't. Microbiol Aust. 2020. https://doi.org/10.1071/MA20013

3. Special Expert Group for Control of the Epidemic of Novel Coronavirus Pneumonia of the Chinese Preventive Medicine Association. [An update on the epidemiological characteristics of novel coronavirus pneumonia (COVID-19)]. Zhonghua Liu Xing Bing Xue Za Zhi. 2020;41(2):139-144. (In Chinese). https://doi.org/10.3760/cma.j.issn.0254-

6450.2020.02.002.

4. Kwok YLA, Gralton J, McLaws M-L. Face touching: a frequent habit that has implications for hand hygiene. Am J Infect Control 2015;43(2):112-4. https://doi.org/10.1016/j.ajic.2014.10.015.

5. Zhou M, Zhang X, Qu J. Coronavirus disease 2019 (COVID-19): a clinical update. Front Med. 2020;14(2):126-35. https://doi.org/10.1007/s11684-020$0767-8$

6. Baig AM, Khaleeq A. Evidence of COVID -19 virus targeting the CNS: tissue distribution, host virus interaction and proposed neurotropic mechanism. ACS chem. Nuerosceince 2020; 11(7):995-8. https://doi.org/10.1021/acschemneuro.ocoo122

7. YC Li, Bai WZ. The nueroinvasive potential of SARS $\mathrm{Co}-\mathrm{V}$ may play a role in respiratory failure of COVID-19 patients. J Med Virol. 2020;92(6):552-5. https://doi.org/10.1002/jmv.25728

8. Biadsee A, Biadsee A, Kassem F, Dagan O, Masarwa S, Ormianer Z. Olfactory and Oral Manifestations of COVID-19: Sex-Related Symptoms-A Potential Pathway to Early Diagnosis. Otolaryngology-Head and Neck Surgery. 2020;163(4):722-8. https://doi.org/10.1177/0194599820934380

9. Behzad I, Maryam K, Rezvan A, Hamed Z, Mahin A. Oral manifestations of COVID -19 disease: A review article. Dermatologic Therapy 2020; 34(2): e14578. https://doi.org/10.1111/dth.14578.

10. Mullol J, Alobid I, Mariño-Sánchez F, IzquierdoDomínguez A, Marin C, Klimek L, et al. The Loss of Smell and Taste in the COVID-19 Outbreak: a Tale of Many Countries. Curr Allergy Asthma Rep. 2020; 20(10): 61. https://doi.org/10.1007/s11882-020-00961-1

11. Pedrosa MS, Sipert CR, Nogueira FN. Altered taste in patients with COVID-19: The potential role of salivary glands. Oral Dis. 2020: 00:1-3. https://doi.org/10.1111/odi.13496.

12. Dinesh J, Ezhilarasan K, Silas D, Balaji N, Hemalatha $\mathrm{R}$, Padmini S, et al. COVID-19: Poor outcomes in patients with Zinc deficiency. International Journal of 
Infectious Diseases 2020:100:343-9. https://doi.org/10.1016/j.ijid.2020.09.014.

13. Chambers JP, Yu J, Valdes JJ, Arulanandam BP. SARSCoV-2, Early Entry Events. Journal of Pathogens 2020;2020:9238696.

https://doi.org/10.1155/2020/9238696
14. Xu J, Li Y, Gan F, Du Y, Yao Y. Salivary Glands: Potential Reservoirs for COVID-19 Asymptomatic Infection. Journal of Dental Research. 2020;99(8):989. https://doi.org/10.1177/0022034520918518
Cite this article as:

Sangha R, Sumanjit, Sandhu R, Bansal M, Simran. COVID -19: Oral Manifestations-A Dentist's Perspective. Int Healthc Res J. 2021;5(2):RV1-RV5.

\section{AUTHOR AFFILIATIONS: (*Corresponding Author)}

1. Professor, Department of Prosthodontics, Rayat Bahra Dental College and Hospital, Mohali, Punjab, India

2. Senior Lecturer, Department of Prosthodontics, Rayat Bahra Dental College and Hospital, Mohali, Punjab, India

(https://orcid.org/oooo-0003-0419-860o)

3. Senior Lecturer, Department of Prosthodontics, Rayat Bahra Dental College and Hospital, Mohali, Punjab, India

4. MDS, Public Health Dentistry, Private Practitioner, Panchkula, Haryana, India

(https://orcid.org/oooo-ooo1-5522-1351)

5. Tutor, Department of Prosthodontics, Rayat Bahra Dental College and Hospital, Mohali, Punjab, India

(https://orcid.org/oooo-0oo1-6341-2449) 